\title{
Immune Checkpoint Inhibitors for Unresectable Hepatocellular Carcinoma
}

\author{
Mohamed A. Abd El Aziz 1®i , Antonio Facciorusso 2 ${ }^{1}$, Tarek Nayfeh ${ }^{3}$, Samer Saadi ${ }^{3}$, \\ Mohamed Elnaggar ${ }^{4}$, Christian Cotsoglou ${ }^{5}$ and Rodolfo Sacco ${ }^{2,6, * \text { (D) }}$ \\ 1 Department of Surgery, Mayo Clinic, Rochester, MN 55905, USA; abdelmaksoud.mohamed@mayo.edu \\ 2 Gastroenterology Unit, Department of Medical Sciences, Ospedali Riuniti di Foggia, 71122 Foggia, Italy; \\ antonio.facciorusso@virgilio.it \\ 3 Robert D. and Patricia E. Kern Center for the Science of Health Care Delivery, Mayo Clinic, Rochester, \\ MN 55905, USA; Nayfeh.tarek@mayo.edu (T.N.); MohirSaadi.Samer@mayo.edu (S.S.) \\ 4 Department of Internal Medicine, Reno School of Medicine, University of Nevada, Las Vegas, NV 1155, USA; \\ melnaggar@med.unr.edu \\ 5 General Surgery Department, ASST-Vimercate, 20871 Vimercate, Italy; christian.cotsoglou@asst-vimercate.it \\ 6 Gastroenterology Unit, Department of Medical Sciences, Ospedali Riuniti di Foggia, Viale Pinto, 1, \\ 71100 Foggia, Italy \\ * Correspondence: r.sacco@ao-pisa.toscana.it
}

Received: 14 September 2020; Accepted: 14 October 2020; Published: 19 October 2020

\begin{abstract}
Despite the advances in screening protocols and treatment options, hepatocellular carcinoma (HCC) is still considered to be the most lethal malignancy in patients with liver cirrhosis. Moreover, the survival outcomes after failure of first-line therapy for unresectable HCC is still poor with limited therapeutic options. One of these options is immune checkpoint inhibitors. The aim of this study is to comprehensively review the efficacy and safety of immune checkpoint inhibitors for patients with HCC.
\end{abstract}

Keywords: HCC; CPI; immunotherapy; survival; progression

\section{Introduction}

Hepatocellular carcinoma (HCC) is still the most common and most lethal malignancy in patients with liver cirrhosis, despite the advances in screening programs, chemoprophylaxis for high-risk patients and treatment options [1,2]. With the rapid increase in prevalence of metabolic disorders, nonalcoholic fatty liver disease became one of the leading risk factors of HCC after hepatitis B and C $[3,4]$. Overall, HCC is considered an inflammatory prototypic cancer. The high mortality rate from $\mathrm{HCC}$ is related to late diagnosis and the concomitant liver dysfunction. In that case, usually, curative resection or liver transplantation is not feasible [5].

Despite the recent advances in systemic therapy for unresectable HCC, patients who progress on first-line multikinase inhibitors, namely sorafenib [which targets vascular endothelial growth factor receptor (VEGFR), platelet-derived growth factor receptor (PDGFR- $\beta$ ) and rapidly accelerated fibrosarcoma (RAF) kinases] [6-8] and lenvatinib (which targets VEGFR1, VEGFR2 and VEGFR3, PDGFR alpha, fibroblast growth factor receptor (FGFR) and KIT and RET tyrosine kinases) [9-13], have limited options [5,14]. Moreover, these systemic therapies are usually associated with significant resistance and side-effects. Furthermore, some clinical trials designed to expand on the already existing options for patients with HCC showed disappointing results [15]. However, recently four additional targeted therapies got approval for treatment of HCC based on phase III randomized controlled trials. Those therapies include lenvatinib as first-line therapy [9] and regorafenib [14,16], cabozantinib [17] and ramucirumab [18] as rescue therapies after failure of sorafenib. 
The tumor microenvironment of the HCC is infiltrated with different types of immune cells, mainly T-cells (CD8+, CD4+, Treg), natural killer cells and myeloid cells (myeloid-derived suppressor cells and tumor-associated macrophages). Due to the chronic inflammation and cirrhosis present in most HCC patients, the tumor ecosystem gets complicated affecting the behavior of the tumor and response to treatment. These changes are due to complex interactions between immune cells and tumor cells in the tumor microenvironment conveyed through cytokines and signaling pathways leading to exhaustion of pro-inflammatory immune cells and the dominance of the regulatory leukocytes hindering the anti-tumor response. A study by Yu et al. [19] concluded that improved overall survival was associated with high immune infiltration. The study further identified different immune clusters based on their prognostic value showing that better outcomes were associated with clusters with high levels of T-cells (mainly CD8+) and low levels of macrophages. A subset of tumor-associated macrophages (M1) was shown to be associated with improved outcomes. Poor prognosis is associated with the accumulation of myeloid-derived suppressor cells, tumor-associated macrophages, CD4+/CD25+/FOXP3+ immune-suppressive T-cells(T-reg), exhausted Th1 CD4+, CD8+ T-cells, dysfunctional NK cells and the expansion of Th2 CD4+ T-cells. Immune checkpoint molecules including programmed cell death (PD-1), CD274, cytotoxic T lymphocyte antigen -4 (CTLA-4), lymphocyte activated gene -3 (LAG-3) and IFNG were identified in clusters that had high levels of CD8+ T-cells. However, these clusters were associated with poor prognosis which leads to the assumption that these molecules are implicated in the HCC immune-exhaustion [20]. Therefore, it is assumed that the administration of immune checkpoint inhibitors would be beneficial for these HCC patients. In the United States, accelerated approval has been granted by the Food and Drug Administration (FDA) to two anti-programmed cell death monoclonal antibodies (nivolumab and pembrolizumab) and a combination of nivolumab plus ipilimumab, a monoclonal antibody against CTLA-4, for patients who progressed on sorafenib based on the results of several phase III trials [21-23]. However, data from phase III trial did not show superior efficacy of nivolumab as first-line therapy over sorafenib [24]. Moreover, the results of KEYNOTE 240 which assessed pembrolizumab as second-line therapy compared to placebo did not meet its predetermined level of statistical significance [25]. Therefore, we aimed to review the current evidence in the literature regarding the use of immune checkpoint inhibitors for the treatment of HCC.

\section{Immune Checkpoint Inhibitors as a First-Line Therapy}

\subsection{PD-1/PD-L1 Inhibition}

Avoiding immune destruction is one of the hallmarks of cancer. The PD-1/PD-L1 pathway plays a pivotal role in this escape mechanism [26]. Studies have shown that PD-L1 is overexpressed in tumor cells in different types of cancers including HCC, which leads to an increase in binding between PD-L1 and PD-1 on T cells within the tumor microenvironment resulting in immune anergy and apoptosis $[27,28]$. As a result, with overexpression of PD-L1, the tumor continues to grow unchecked which leads to worse prognosis in patients with HCC [28,29]. Interfering with this binding can result in enhancing immune reaction toward the cancer cells. (Figure 1) Therefore, the introduction of monoclonal antibody in the landscape of treatment of HCC has gained accelerated approval for patients who previously progressed on sorafenib based on the results CheckMate 040 trail [21]. However, for first-line therapy, the CheckMate 459 trial compared nivolumab to sorafenib in patients with Child-Pugh A (non-severe liver cirrhosis). Although the objective response rate was higher in the nivolumab group than the sorafenib group, the overall survival and progression-free survival were not significantly different between both groups [24] (Table 1). 
Table 1. Immune checkpoint inhibitors as first-line therapy for unresectable hepatocellular carcinoma (HCC).

\begin{tabular}{|c|c|c|c|c|c|c|c|}
\hline Study ID & NCT & $\begin{array}{l}\text { Study Design, } \\
\text { Key Inclusion }\end{array}$ & Sample Size & OS, Months $(95 \%$ CI) & PFS Months $(95 \% \mathrm{CI})$ & Response Rates & Side Effects \\
\hline \multicolumn{8}{|c|}{ PD-1/PD-L1 antibodies } \\
\hline $\begin{array}{c}\text { CheckMate } 459 \\
\text { ESMO October } 2019\end{array}$ & NCT02576509 & RCT, CP: A & $\begin{array}{l}743 \text { patients } \\
\text { Nivolumab: } 371 \mathrm{pts} \\
\text { Sorafenib: } 372 \mathrm{pts}\end{array}$ & $\begin{array}{c}\text { Nivolumab vs. Sorafenib: } \\
\text { OS: } \\
16.4(13.9-18.4) \text { vs. } \\
14.7(11.9-17.2) \\
12 \mathrm{mo}(\%): \\
59.7(54.4-64.6) \text { vs. } \\
55.1(49.8-60.1) \\
24 \mathrm{mo}(\%): \\
36.8(31.8-41.8) \text { vs. } \\
33.1(28.3-38.0)\end{array}$ & $\begin{array}{c}\text { Nivolumab vs. Sorafenib: } \\
3.7(3.1-3.9) \text { vs. } \\
3.8(3.7-4.5)\end{array}$ & $\begin{array}{l}\text { Nivolumab vs. Sorafenib: } \\
\text { ORR: } 57(15 \%) \text { vs. } 26(7 \%) \\
\text { Complete response: } \\
14 \text { (4\%) vs. } 5(1 \%) \\
\text { Partial response: } \\
43(12 \%) \text { vs. } 21(6 \%)\end{array}$ & $\begin{array}{l}\text { Nivolumab demonstrated a } \\
\text { favorable safety profile } \\
\text { consistent with } \\
\text { previous reports. }\end{array}$ \\
\hline RATIONALE 301 & NCT03412773 & $\begin{array}{l}\text { RCT, BCLC stage } \\
\text { Cor B, CP: A }\end{array}$ & \begin{tabular}{c|}
674 patients \\
Tislelizumab vs. Sorafenib
\end{tabular} & Pending & Pending & Pending & Pending \\
\hline \multicolumn{8}{|c|}{ Dual immune checkpoint blockade: } \\
\hline HIMALAYA study & NCT03298451 & $\begin{array}{c}\text { RCT, } \\
\text { BCLC stage } \mathrm{C} \text { or } \mathrm{B}, \\
\text { CP: A }\end{array}$ & $\begin{array}{c}1310 \mathrm{pts} \\
\text { Durvalumab vs. } \\
\text { (Durvalumab + Tremelimumab) } \\
\text { vs. } \\
\text { Sorafenib }\end{array}$ & Pending & Pending & Pending & Pending \\
\hline \multicolumn{8}{|c|}{ Combination with biological therapy: } \\
\hline IMbrave 150 & NCT03434379 & $\begin{array}{l}\mathrm{RCT}, \\
\mathrm{CP}: \mathrm{A}\end{array}$ & $\begin{array}{c}501 \text { patients } \\
\text { Atezolizumab + Bevacizumab: } \\
336 \text { pts vs. } \\
\text { Sorafenib: } 165 \text { pts }\end{array}$ & $\begin{array}{c}\text { Atezolizumab + Bevacizumab } \\
\text { vs. Sorafenib; } \\
\text { Overall death: } \\
\text { 28.6\% vs. } 39.4 \% ; \mathrm{HR}: 0.58 \\
\text { (95\% CI } 0.42-0.79) \\
\text { OS: } \\
\text { NE vs. } 13.2(10.4-\mathrm{NE}) \\
\text { OS at } 6 \text { Mo: } \\
84.8 \% \text { vs. } 72.2 \%\end{array}$ & $\begin{array}{c}\text { Atezolizumab + Bevacizumab } \\
\text { vs. Sorafenib; } \\
\text { Overall progression: } \\
\text { 58.6\% vs. 66.1\%; HR: } 0.59 \\
\text { (95\% CI 0.47-0.76) } \\
\text { PFS: } \\
\text { 6.8 (5.7-8.3) vs. } 4.3(4.0-5.6) \\
\text { PFS at } 6 \text { Mo: } \\
\text { 57.5\% vs. } 37.2 \%\end{array}$ & $\begin{array}{c}\text { Atezolizumab + Bevacizumab } \\
\text { vs. Sorafenib; } \% \text { (95\% CI) } \\
\text { ORR per RECIST 1.1: } \\
\text { 27.3 (22.5-32.5) vs. } \\
11.9 \text { (7.4-18) } \\
\text { ORR per HCC specific } \\
\text { mRECIST: } \\
\text { 33.2 (28.1-38.6) vs. } \\
13.3(8.4-19.6)\end{array}$ & $\begin{array}{c}\text { Atezolizumab }+ \\
\text { Bevacizumab vs. Sorafenib; } \\
\text { Grade 3-4 complications: } \\
186(56.5 \%) \text { vs. } 86(55.1 \%)\end{array}$ \\
\hline
\end{tabular}


Table 1. Cont

\begin{tabular}{|c|c|c|c|c|c|c|c|}
\hline Study ID & NCT & $\begin{array}{l}\text { Study Design, } \\
\text { Key Inclusion }\end{array}$ & Sample Size & OS, Months $(95 \% \mathrm{CI})$ & PFS Months ( $95 \% \mathrm{CI})$ & Response Rates & Side Effects \\
\hline \multicolumn{8}{|c|}{ Combination with biological therapy: } \\
\hline G030140 group F & NCT02715531 & $\mathrm{RCT}, \mathrm{CP}: \mathrm{A}$ & $\begin{array}{c}119 \mathrm{pts} \\
\text { Atezolizumab + Bevacizumab: } \\
60 \mathrm{pts} \text { vs. } \\
\text { Atezolizumab: } 59 \mathrm{pts}\end{array}$ & $\begin{array}{l}\text { Atezolizumab + Bevacizumab } \\
\text { vs. Atezolizumab } \\
\text { Overall death: } \\
27 \% \text { vs. } 31 \% \\
\text { OS: not reached in both groups }\end{array}$ & $\begin{array}{c}\text { Atezolizumab + Bevacizumab } \\
\text { vs. Atezolizumab } \\
\text { Overall progression: } \\
\text { HR: } \\
\text { per HCC } m \text { RECIST: } \\
57 \% \text { vs. 66\%, } \\
\text { HR: } 0.54 \text { ( } 80 \% \text { CI } 0.40-0.74) \\
\text { per RECIST 1.1: } \\
58 \% \text { vs. } 66 \% \\
\text { HR: } 0.55 \text { (80\% CI } 0.40-0.74) \\
\text { PFS Mo: } \\
\text { per HCC mRECIST: } \\
5.6 \text { mo (3.6-7.4) vs. } 3.4 \text { mo } \\
(1.9-5.2) \\
\text { per RECIST: } \\
5.7 \text { mo (3.5-9.3) vs. } 2.0 \text { mo } \\
(1.9-3.7)\end{array}$ & $\begin{array}{c}\text { Atezolizumab + Bevacizumab } \\
\text { vs. Atezolizumab } \\
\text { ORR per RECIST 1.1: } \\
\text { 20\% (95\% CI 11-32) vs. } 17 \% \\
\text { (95\% CI 8-29) } \\
\text { ORR per HCC mRECIST: } \\
27 \% \text { (95\% CI 16-40) vs. } 17 \% \\
\text { (95\% CI 8-29) }\end{array}$ & $\begin{array}{c}\text { Atezolizumab + } \\
\text { Bevacizumab vs. } \\
\text { Atezolizumab } \\
\text { Grade 3-4: } \\
12 \text { (20\%) vs. 3 }(5 \%) \\
\text { The most common grade } \\
\text { 3-4 SEs were: hypertension: } \\
\text { 3 }(5 \%) \text { vs. none } \\
\text { proteinuria: } \\
2(3 \%) \text { vs. none }\end{array}$ \\
\hline G030140 group A & NCT02715531 & $\mathrm{RCT}, \mathrm{CP}: \mathrm{A}$ & $\begin{array}{c}104 \text { pts } \\
\text { Atezolizumab + Bevacizumab }\end{array}$ & $\begin{array}{l}57(55 \%) \text { still alive at data cut off } \\
\text { OS not reached }\end{array}$ & $\begin{array}{c}\text { Per RECIST 1.1: } \\
\text { 66\%; 7.3 months ( } 95 \% \text { CI } \\
5.4-9.9) \\
\text { Per HCC mRECIST: } \\
66 \% ; 7.3 \text { months }(95 \% \text { CI } \\
5.4-9.9)\end{array}$ & $\begin{array}{c}\text { ORR per RECIST 1.1: n (\%; } \\
95 \% \text { CI) } \\
37(36 \% ; 26-46) \\
\text { ORR per HCC mRECIST: } \\
41(39 \% ; 30-50)\end{array}$ & $\begin{array}{c}\text { Serious SEs: } 25(24 \%) \\
\text { The most common serious } \\
\text { SEs were upper } \\
\text { gastrointestinal } \\
\text { hemorrhage, colitis, } \\
\text { esophageal variceal } \\
\text { hemorrhage and } \\
\text { pneumonitis, each occurring } \\
\text { in two }(2 \%) \text { patients. }\end{array}$ \\
\hline COSMIC 312 & NCT03755791 & $\begin{array}{l}\text { RCT, BCLC stage } \\
\text { C or B, CP: A }\end{array}$ & $\begin{array}{c}740 \text { pts } \\
\text { Cabozantinib + Atezolizumab: } \\
370 \text { pts vs. } \\
\text { Cabozantinib: } 185 \text { pts vs. } \\
\text { Sorafenib: } 185 \text { pts }\end{array}$ & pending & pending & pending & Pending \\
\hline LEAP 002 & NCT03713593 & $\begin{array}{l}\text { RCT, BCLC stage } \\
\text { C or B, CP: A }\end{array}$ & $\begin{array}{c}750 \mathrm{pts} \\
\text { Pembrolizumab + Lenvatinib } \\
\text { vs. } \\
\text { Lenvatinib alone }\end{array}$ & pending & pending & pending & Pending \\
\hline CheckMate 9DW & NCT04039607 & RCT & $\begin{array}{c}1084 \text { pts } \\
\text { Nivolumab + Ipilimumab vs. } \\
\text { Sorafenib/Lenvatinib }\end{array}$ & pending & pending & pending & Pending \\
\hline
\end{tabular}


Table 1. Cont.

\begin{tabular}{|c|c|c|c|c|c|c|c|}
\hline Study ID & NCT & $\begin{array}{l}\text { Study Design, } \\
\text { Key Inclusion }\end{array}$ & Sample Size & OS, Months $(95 \%$ CI) & PFS Months $(95 \% \mathrm{CI})$ & Response Rates & Side Effects \\
\hline \multicolumn{8}{|c|}{ Combination with biological therapy: } \\
\hline $\begin{array}{l}\text { KEYNOTE 524; } \\
\text { AACR April } 2019\end{array}$ & NCT03006926 & $\begin{array}{l}\text { Single-arm, BCLC } \\
\text { stage C or B, CP: A }\end{array}$ & $\begin{array}{l}104 \text { pts will be recruited, } \\
\text { however, the presented results } \\
\text { are for } 30 \text { pts ( } 6 \text { pts in safety } \\
\text { part and } 24 \text { pts in efficacy part) } \\
\text { Pembrolizumab + Lenvatinib }\end{array}$ & pending & pending & $\begin{array}{c}\text { ORR } \\
\text { per mRECIST per investigator: } \\
11(36.7) \\
\text { per mRECIST per IIR: } \\
15(50.0 \%) \\
\text { Per RECIST IIR: } \\
11(36.7 \%)\end{array}$ & $\begin{array}{c}\text { Any-grade } \\
\text { treatment-emergent adverse } \\
\text { events (TEAEs) occurred in } \\
28 \text { pts }(93 \%) \text {; the most } \\
\text { common any-grade TEAEs } \\
\text { were decreased appetite } \\
(63 \%) \text { and hypertension } \\
(60 \%) .7(23 \%) \text { pts } \\
\text { discontinued treatment due } \\
\text { to TEAEs and no new safety } \\
\text { signals were identified. }\end{array}$ \\
\hline VEGF Liver 100 & NCT03289533 & $\begin{array}{l}\text { Single-arm, BCLC } \\
\text { stage C or B, CP: A }\end{array}$ & $\begin{array}{c}22 \mathrm{pts} \\
\text { Avelumab + Axitinib }\end{array}$ & - & $\begin{array}{c}\text { PFS: mo (95\% CI) } \\
\text { Per RECIST: } \\
5.5(1.9-7.3) \\
\text { Per mRECIST: } \\
3.8(1.9-7.3) \\
\text { 6 months PFS: } \%(95 \% \mathrm{CI}) \\
\text { Per RECIST: } \\
35.1 \%(15.3-55.8 \%) \\
\text { Per mRECIST: } \\
30.9 \%(12.5-51.5 \%)\end{array}$ & $\begin{array}{c}\text { ORR } \\
\text { Per RECIST: } \\
\text { 13.6\% (95\% CI, } 2.9-34.9 \%) \\
\text { Per mRECIST: } \\
31.8 \%(95 \% \text { CI } 13.9-54.9 \%)\end{array}$ & $\begin{array}{l}\text { The most common grade } 3 \\
\text { treatment-related adverse } \\
\text { events (TRAEs) }(\geq 10 \% \text { of } \\
\text { patients) were hypertension } \\
\text { (50.0\%) and hand-foot } \\
\text { syndrome (22.7\%); ;o grade } \\
\text { 4/5 TRAEs were reported. }\end{array}$ \\
\hline Kelley 2017, arm five & NCT02519348 & RCT & $\begin{array}{c}433 \text { pts } \\
\text { Durvalumab + Tremelimumab } \\
\text { vs. } \\
\text { Durvalumab vs. } \\
\text { Tremelimumab vs. } \\
\text { Durvalumab + Tremelimumab } \\
\text { (regmine two) vs. } \\
\text { Durvalumab + Bevacizumab }\end{array}$ & - & - & - & - \\
\hline
\end{tabular}

CP: Child-Pugh, RCT: Randomized Controlled Trial, RECIST: Response Evaluation Criteria in Solid Tumors, OS: Overall Survival, PFS: Progression-Free Survival, ORR: Objective Response Rate. 


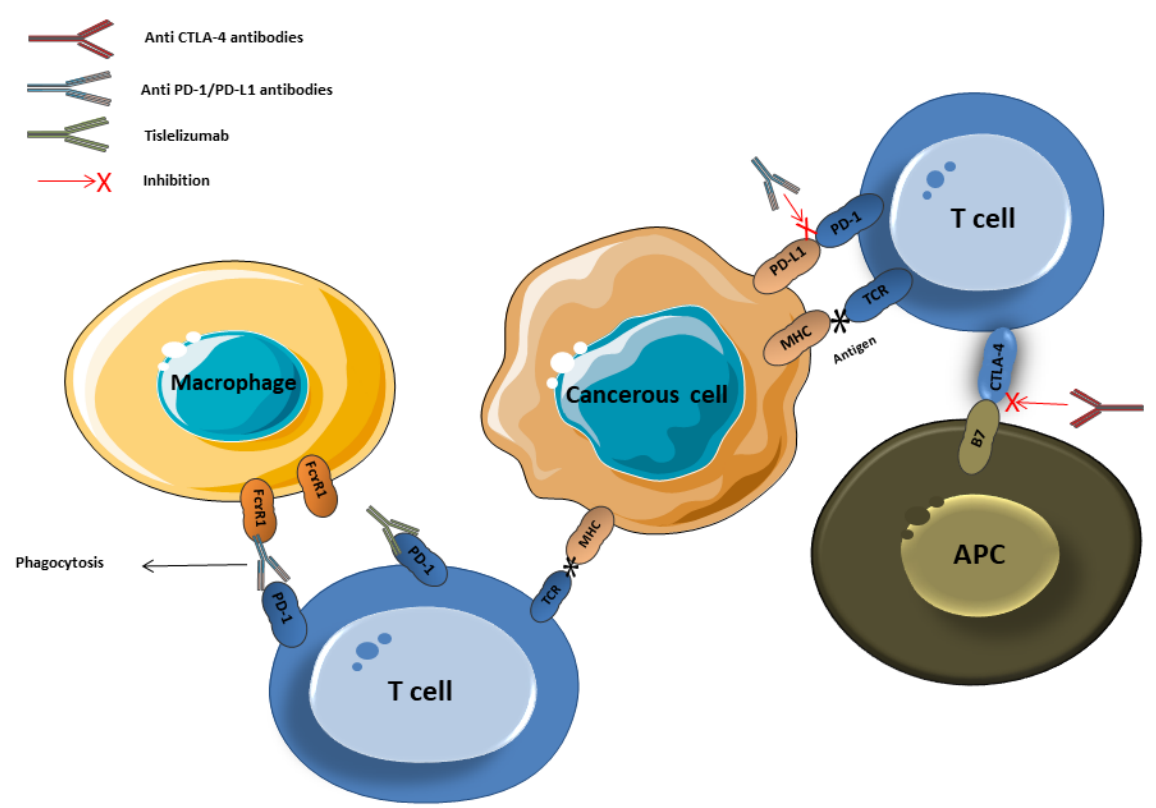

Figure 1. Immune checkpoints inhibitors' mechanism of action. PD: programmed cell death, CTLA-4: cytotoxic T-lymphocyte associated protein 4, APC: antigen-presenting cell, MHC: major histocompatibility complex, TCR: T cell.

One of the well-known mechanisms of resistance to anti-PD-1 therapy is Fc $\gamma$ R1 mediated macrophage antibody-dependent phagocytosis [30] (Figure 1). Therefore, another monoclonal antibody has been developed to evade the Fc $\gamma$ R1 mediated resistance that is, tislelizumab [31]. Clinical data from the RATIONAL 301 trial, which is comparing tislelizumab against sorafenib, supporting this mechanism are still pending [31].

\subsection{Dual Immune Checkpoint Blockade}

CTLA-4 is expressed on T regulatory cells regulating the early immune response after the primary stimulation by antigens mainly in lymphoid organs whereas PD-1 is expressed mainly on activated T cells in the tumor microenvironment regulating late immune response. Moreover, inhibition of the CTLA-4/B7 signal in lymph nodes increases activated CD8+ cells which will subsequently infiltrate the tumor and be part of the microenvironment $[32,33]$. Based on this, several studies have shown promising results with dual immunotherapy $[34,35]$. The success achieved in these trials especially in for patients with melanoma [36] has inspired the application of dual immune blockage for other types of cancers including HCC. Therefore, after the success achieved by the phase I/II trial investigating the efficacy and safety of dual immune therapy for patients progressed on sorafenib [37], a comparative randomized controlled trial, HIMALAYA study, was designed to compare Duravalumab versus the combination of Duravalumab plus Tremelimumab versus sorafenib. Its results are still bending (Table 1).

\subsection{Combination with Biological Therapy}

Vascular endothelial growth factor (VEGF) has been linked with the development and progression of HCC $[38,39]$. Moreover, it has a role in immune suppression as it has been found that it creates an immunosuppressive microenvironment through the recruitment of several inhibitory cells such as $\mathrm{T}$ regulatory cells, tumor-associated macrophages and myeloid-derived suppressor cells. Those cells release cytokines such as IL-10 and TGF- $\beta$ that inhibit natural killer cell and T cell activation and impedes dendritic cell maturation as shown in Figure 2 [33,40,41].

The landmark IMbrave 150 trial comparing atezolizumab (PD-L1 monoclonal antibody) plus bevacizumab (a monoclonal antibody against vascular endothelial growth factor) versus sorafenib found 
a better objective response rate and survival for patients treated with the combination therapy [42]. Moreover, the combination of atezolizumab plus bevacizumab showed a better progression-free survival when compared to atezolizumab alone [43]. As the main concern for patients with liver cirrhosis treated with bevacizumab is upper gastrointestinal bleeding, it occurred in $7 \%$ of the patients who received the combination therapy which is comparable to earlier reports evaluating bevacizumab alone in patients with HCC [42,44,45]. However, proteinuria and hypertension, as main side effects of bevacizumab, still among the top side effects of combination therapy. However, further evaluation of combination therapy versus sorafenib or lenvatinib as first-line therapy for HCC is still under investigation. For example, the combination of nivolumab plus ipilimumab versus sorafenib/lenvatinib as first-line therapy for HCC is still under investigation by the CheckMate 9DW trial (NCT04039607), the combination of cabozantinib plus atezolizumab versus sorafenib is under investigation by COSMIC 312 trial (NCT03755791) and the combination between pembrolizumab plus lenvatinib versus lenvatinib alone is under investigation by the LEAP 002 trial (NCT03713593). Nevertheless, the success achieved by the landmark IMbrave 150 trial and G030140 trial has a great implication for the practice regarding the upfront therapy for patients with unresectable HCC. Nevertheless, these trials included only patients with early liver disease and the efficacy and safety of the combination therapy in patients with advanced liver disease is still unelucidated. Furthermore, no data available about subsequent therapy after the failure of immune checkpoint. More details are provided in Table 1.

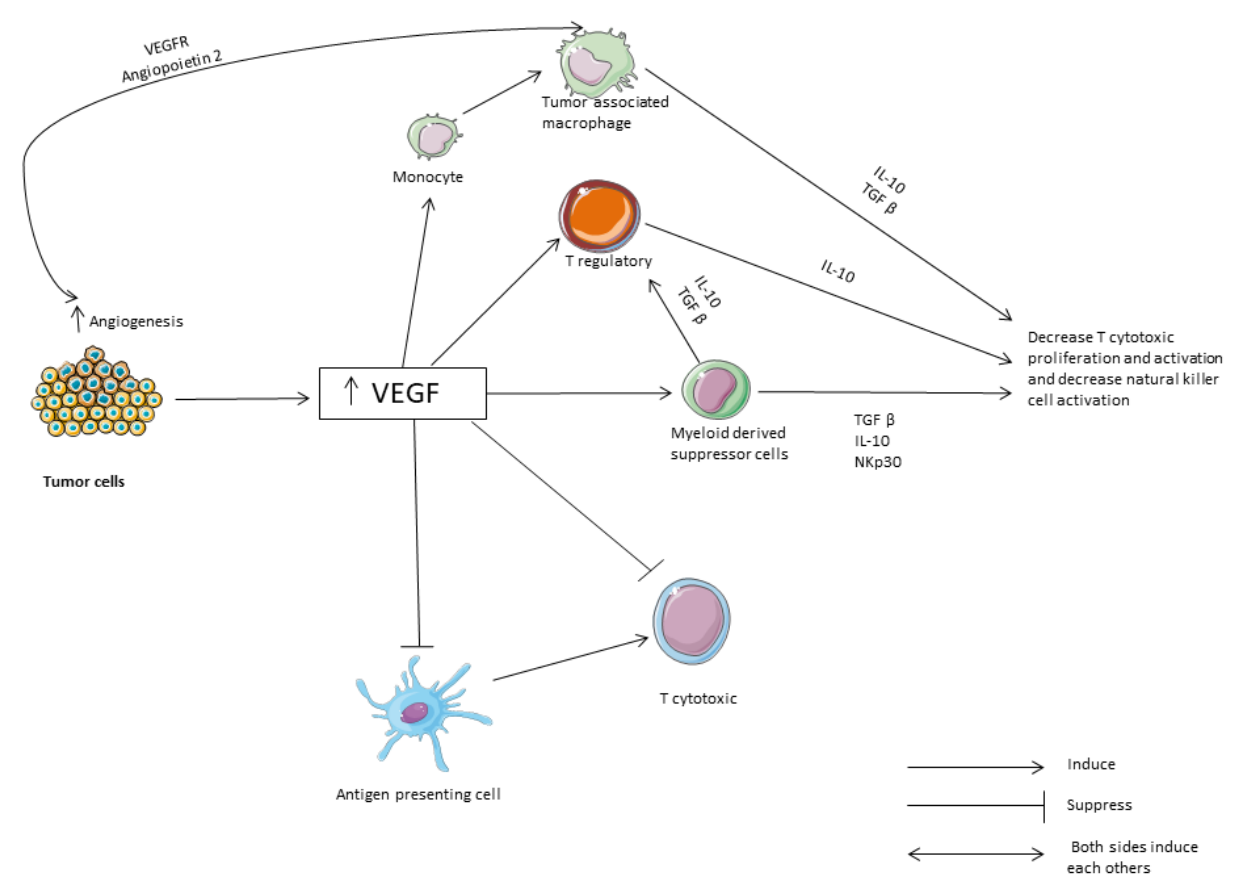

Figure 2. The role of VEGF and cytokines in immune suppression. VEGF: vascular endothelial growth factor. TGF: transformation growth factor.

\section{Immune Checkpoint Inhibitors as Second-Line Therapy}

\subsection{CTLA-4 Inhibition}

Sangro et al. recruited 21 patients with hepatitis $C$ virus who had progressed on previous lines of treatment for HCC. The treatment was tremelimumab at a dose of $15 \mathrm{mg} / \mathrm{kg}$ IV every 90 days. The drug showed a safe profile with a partial response rate of $17.6 \%$ [46]. Interestingly, the viral load for HCV decreased. Denoting the antiviral effect with the enhanced immunity. Moreover, the addition of ablation therapy to the anti-CTLA-4 showed a higher response rate with a similar safety profile [47] (Table 2). 
Table 2. Immune checkpoint inhibitors after failure or intolerability for first-line therapy for patients with unresectable HCC.

\begin{tabular}{|c|c|c|c|c|c|c|c|}
\hline Study ID & NCT & $\begin{array}{l}\text { Study Design, Key } \\
\text { Inclusion }\end{array}$ & Sample Size & OS, Months ( $95 \% \mathrm{CI})$ & PFS Months (95\% CI) & Response Rates & Side Effects \\
\hline \multicolumn{8}{|c|}{ CTLA-4 antibodies } \\
\hline Sangro 2013 & NCT01008358 & $\begin{array}{c}\text { Single-arm, HCV } \\
\text { patients, CP: A or B }\end{array}$ & $\begin{array}{c}21 \mathrm{pts} \\
\text { Tremelimumab }\end{array}$ & - & - & $\begin{array}{c}\text { ORR: } 17.6 \% \\
\text { time to progression: } 6.48 \\
\text { months (95\% CI 3.95-9.14) }\end{array}$ & $\begin{array}{c}\text { Grade } 3-4 \text { transaminase } \\
\text { elevation: } 45 \%\end{array}$ \\
\hline Duffy 2017 & NCT01853618 & Single-arm, CP: A or B & $\begin{array}{l}32 \text { pts } \\
\text { Tremelimumab } \\
\text { plus ablation }\end{array}$ & $\begin{array}{c}\text { OS: } 12.3 \text { months ( } 95 \% \text { CI } 9.3 \\
\text { to } 15.4 \text { months). } \\
\text { Six months OS: } 85.7 \% \\
\text { (66.3-94.4\%) } \\
\text { One year OS: } 50.8 \% \\
(29.1-68.9 \%)\end{array}$ & $\begin{array}{c}\text { PFS: } 7.4 \text { months } \\
\text { (4.7-19.4 months) } \\
\text { Six months PFS: } 57.1 \% \\
\text { (37.1-72.9\%) } \\
\text { One year PFS: } 33.1 \% \\
(16.2-51.2 \%)\end{array}$ & $\begin{array}{l}\text { Partial response: } 26 \% \\
\quad(95 \% \text { CI } 9.1-51.2)\end{array}$ & $\begin{array}{c}\text { Grade } 3-4 \text { increase AST } \\
7 \text { pts }(19 \%)\end{array}$ \\
\hline \multicolumn{8}{|c|}{ PD-1/PD-L1 inhibition: } \\
\hline KEYNOTE 240 & NCT02702401 & $\mathrm{RCT}, \mathrm{CP}: \mathrm{A}$ & $\begin{array}{c}413 \text { pts } \\
\text { Pembrolizumab: } 278 \text { pts vs. } \\
\text { Placebo: } 135 \text { pts }\end{array}$ & $\begin{array}{c}\text { OS: } \\
\text { Pembrolizumab: } 13.9 \\
\text { months (95\% CI, } 11.6 \text { to } \\
16.0 \text { months) } \\
\text { Placebo: } 10.6 \text { months } \\
\text { (95\% CI, } 8.3 \text { to } 13.5 \text { months) } \\
\text { HR: } 0.781 ; 95 \% \text { CI, } 0.611 \\
\text { to } 0.998\end{array}$ & $\begin{array}{c}\text { PFS: } \\
\text { Pembrolizumab: } 3.0 \text { months } \\
\text { (95\% CI, } 2.8 \text { to } 4.1 \text { months) } \\
\text { Placebo: } 2.8 \text { months } \\
\text { (95\% CI, } 1.6 \text { to } 3.0 \text { months) } \\
\text { HR: } 0.718 ; 95 \% \text { CI, } \\
0.570 \text { to } 0.904 \\
\text { PFS at } 12 \text { months: } \\
\text { Pembrolizumab: } 19.4 \% \\
\text { (95\% CI, } 14.6 \% \text { to } 24.9 \%) \\
\text { Placebo: } 6.7 \%(95 \% \text { CI, } \\
\text { 3.0\% to } 12.4 \%)\end{array}$ & $\begin{array}{c}\text { ORR: } \\
\text { Pembrolizumab: } 18.3 \% \\
\text { (95\% CI 14-23.4) } \\
\text { Placebo: } 4.4 \%(95 \% \text { CI } \\
1.6-9.4) \\
\text { Estimated treatment } \\
\text { difference: } 13.8(95 \% \text { CI: } \\
7.7 \text { to } 19.5)\end{array}$ & $\begin{array}{c}\text { Any grade 3-4: } \\
\text { Pembrolizumab: } 52 \% \\
\text { Placebo: } 46.3 \% \\
\text { Grade 3-4 AST } \\
\text { elevation: } \\
\text { Pembrolizumab: } 13.3 \% \\
\text { Placebo: } 7.5 \%\end{array}$ \\
\hline Scheiner 2019 & NA & Retrospective cohort & $\begin{array}{c}65 \mathrm{pts} \\
\text { Nivolumab: } 34 \mathrm{pts} \\
\text { Pembrolizumab: } 31 \mathrm{pts}\end{array}$ & $\begin{array}{c}\text { OS: } \\
\text { Nivolumab: } 9.0(95 \% \text { CI, } \\
5.5-12.5) \text { months } \\
\text { Pembrolizumab: } 11.0 \\
\text { (95\% CI, 7.4-14.5) months } \\
\text { 1 year OS: } \\
\text { Nivolumab: } 38 \% \\
\text { Pembrolizumab: } 44 \%\end{array}$ & $\begin{array}{c}\text { PFS } \\
\text { Nivolumab: } 4.3(95 \% \mathrm{CI} \text {, } \\
\text { 2.0-6.7) months } \\
\text { Pembrolizumab: } 5.6 \\
(95 \% \text { CI, 1.1-10.1) months }\end{array}$ & $\begin{array}{c}\text { ORR: } \\
\text { Nivolumab: } 15 \% \\
\text { Pembrolizumab: } 10 \%\end{array}$ & $\begin{array}{l}\text { High grade: } \\
17 \% \text { in both groups }\end{array}$ \\
\hline Choi 2020 & NA & $\begin{array}{l}\text { Propensity score } \\
\text { matching, CP: A }\end{array}$ & $\begin{array}{l}272 \text { pts after matching } \\
\text { Regorafenib: } 136 \text { pts vs. } \\
\text { Nivolumab: } 136 \text { pts }\end{array}$ & $\begin{array}{c}\text { weeks, median }(95 \% \text { CI) } \\
\text { Regorafenib: } 31.3(24.6-42.0) \\
\text { Nivolumab: } 37.1(22.4-49.0)\end{array}$ & $\begin{array}{c}\text { time in weeks; median } \\
(95 \% \mathrm{CI}) \\
\text { Regorafenib: } 12.6(10.6-15.7) \\
\text { Nivolumab: } 7.1(6.1-11.1)\end{array}$ & $\begin{array}{l}\text { ORR: } \\
\text { Regorafenib: } 3.7 \% \\
\text { Nivolumab: } 14 \%\end{array}$ & \\
\hline
\end{tabular}


Table 2. Cont

\begin{tabular}{|c|c|c|c|c|c|c|c|}
\hline Study ID & NCT & $\begin{array}{l}\text { Study Design, Key } \\
\text { Inclusion }\end{array}$ & Sample Size & OS, Months ( $95 \% \mathrm{CI})$ & PFS Months ( $95 \%$ CI) & Response Rates & Side Effects \\
\hline \multicolumn{8}{|c|}{ PD-1/PD-L1 inhibition: } \\
\hline Lee 2020 & NA & Retrospective cohort & $\begin{array}{l}150 \text { patients } \\
\text { Regorafenib: } 102 \text { patients } \\
\text { Nivolumab: } 48 \text { patients }\end{array}$ & $\begin{array}{c}\text { OS: } \\
\text { Regorafenib: 6.9 months } \\
\text { (95\% CI, 3.5-13.1) } \\
\text { Nivolumab: 5.9 } \\
\text { months (95\% CI, 3.2-18.1) } \\
\text { Death rates: } \\
\text { Regorafenib: } 37.3 \% \\
\text { Nivolumab: } 56.3 \%\end{array}$ & $\begin{array}{c}\text { mTTP } \\
\text { Regorafenib: 3.3 months; } \\
\text { (95\% CI, 2.0-5.3) } \\
\text { Nivolumab: 4.0 months; } \\
\text { (95\% CI, 1.8-8.7) } \\
\text { Progression: } \\
\text { Regorafenib: } 60.8 \% \\
\text { Nivolumab: } 60.4 \%\end{array}$ & $\begin{array}{l}\text { ORR: } \\
\text { Regorafenib: } 5.9 \% \\
\text { Nivolumab: } 16.7 \%\end{array}$ & \\
\hline Yu 2019 & NA & Retrospective cohort & $\begin{array}{l}76 \text { pts } \\
\text { Nivolumab alone: } 22 \text { pts } \\
\text { Nivolumab plus } \\
\text { radiotherapy: } 54 \text { pts }\end{array}$ & $\begin{array}{l}\text { Patients who had received } \\
\text { previous/concurrent RT had } \\
\text { a significantly } \\
\text { longer progression-free } \\
\text { survival (PFS; } p=0.008) \\
\text { and overall survival (OS; } \\
p=0.007 \text { ) } \\
\text { than those who did not } \\
\text { receive RT }\end{array}$ & - & $\begin{array}{c}\text { No complete response } \\
\text { PR: } \\
\text { Nivolumab alone: } \\
1 \text { pt }(4.5 \%) \\
\text { Nivolumab plus radio: } \\
8 \text { pts }(14.8 \%)\end{array}$ & $\begin{array}{c}\text { Nivolumab-related } \\
\text { toxicities were generally } \\
\text { tolerable regardless of } \\
\text { the history of RT. }\end{array}$ \\
\hline Qin 2020 & NCT02989922 & RCT & $\begin{array}{l}\text { Total } 220 \text { pts } \\
\text { Camrelizumab every two } \\
\text { weeks group: } 111 \text { pts } \\
\text { Camrelizumab every three } \\
\text { weeks group: } 109 \text { pts. }\end{array}$ & $\begin{array}{c}\text { OS: } \\
\text { Overall: } 13.8(11.5-16.6) \\
\text { Two months: } 14.2(11.5-\mathrm{NR}) \\
\text { three months: } 13.2(9.4-17.0) \\
\text { OS rates: } \\
\text { At } 6 \text { months, \% (95\% CI): } \\
\text { Overall: } 74.4 \%(68.0-79.7) \\
\text { Two weeks: } 75.9 \% \\
\text { (66.6-82.9) } \\
\text { Three weeks: 73.0\% } \\
\text { (63.6-80.4) } \\
\text { At } 9 \text { months: } \\
\text { Overall: 64.0\% (57.2-70.1) } \\
\text { Two weeks: 67.3\% } \\
\text { (57.5-75.3) } \\
\text { Three weeks: 60.8\% } \\
\text { (50.8-69.3) } \\
\text { At 12 months: } \\
\text { Overall: 55.9\% (48.9-62.2) } \\
\text { Two weeks: } 59.6 \% \\
\text { (49.6-68.2) } \\
\text { Three weeks:52.2\% } \\
\text { (42.3-61.2) }\end{array}$ & $\begin{array}{c}\text { PFS: } \\
\text { Overall: } 2.1 \text { months (2.0-3.2) } \\
\text { Two weeks: } 2.3 \text { months } \\
\text { (1.9-3.2) } \\
\text { Three weeks: } 2.0 \text { months } \\
\text { (2.0-3.2) } \\
\text { Disease progression rate: } \\
\text { Overall: } 73 \% \\
\text { Two weeks: } 72 \% \\
\text { Three weeks: } 74 \%\end{array}$ & $\begin{array}{c}\text { ORR: Number }(\%, 95 \% \mathrm{CI}) \\
\text { Overall: } 32(14.7 \% ; \\
10.3-20.2) \\
\text { Every two weeks: } 13(11.9 \% \text {; } \\
6.5-19.5) \\
\text { Every three weeks: } 19 \\
(17.6 \% ; 10.9-26.1)\end{array}$ & $\begin{array}{c}\text { Grade 3: } \\
\text { Overall: } 11(5.1 \%) \\
\text { Two weeks: } 11(10.1 \%) \\
\text { Three weeks: } 6(5.6 \%) \\
\text { Grade } 4: \\
\text { Overall: } 5(2.3 \%) \\
\text { Two weeks: zero } \\
\text { Three weeks: zero (I do } \\
\text { not know how both two } \\
\text { weeks and three weeks } \\
\text { are zero but ht overall } \\
\text { is 5) } \\
\text { Grade five: } \\
\text { Overall: } 1(0.5 \%) \text {, two } \\
\text { and three weeks } \\
\text { are zero. }\end{array}$ \\
\hline
\end{tabular}


Table 2. Cont.

\begin{tabular}{|c|c|c|c|c|c|c|c|}
\hline Study ID & NCT & $\begin{array}{l}\text { Study Design, Key } \\
\text { Inclusion }\end{array}$ & Sample Size & OS, Months $(95 \% \mathrm{CI})$ & PFS Months $(95 \% \mathrm{CI})$ & Response Rates & Side Effects \\
\hline \multicolumn{8}{|c|}{ PD-1/PD-L1 inhibition: } \\
\hline CHECKMATE 040 & Dose escalation & Phase I/2 trial & $\begin{array}{c}48 \mathrm{pts} \\
\text { Nivolumab }\end{array}$ & - & - & - & $\begin{array}{c}\text { Treatment-related grade } \\
3-4: 25 \%\end{array}$ \\
\hline & Dose expansion & & $\begin{array}{l}214 \mathrm{pts} \text { Nivolumab } \\
\text { uninfected Sorafenib } \\
\text { untreated/intolerant: } 56 \mathrm{pts} \\
\text { uninfected Sorafenib } \\
\text { progressors: } 57 \mathrm{pts} \\
\text { HCV: } 50 \mathrm{pts} \\
\text { HBV: } 51 \mathrm{pts}\end{array}$ & $\begin{array}{c}\text { OS: not reached } \\
6 \text { months OS: } \\
\text { Overall: } 83 \% \text { (78 to } 88) \\
\text { uninfected } \\
\text { untreated/intolerant: } 89 \% \\
\text { (77 to } 95) \\
\text { uninfected Sorafenib } \\
\text { progressors: } 75 \% \text { (62 to } 85 \text { ) } \\
\text { HCV: } 85 \% \text { (72 to } 93 \text { ) } \\
\text { HBV: } 84 \% \text { (71 to } 92)\end{array}$ & $\begin{array}{c}\text { PFS: } \\
\text { Overall: } 4.0 \text { (2.9 to } 5.4) \\
\text { uninfected } \\
\text { untreated/intolerant: } 5.4 \\
\text { (3.9 to } 8.5) \\
\text { uninfected Sorafenib } \\
\text { progressors: } 4.0(2.6 \text { to } 6.7) \\
\text { HCV: } 4.0(2.6 \text { to } 5.7) \\
\text { HBV: } 4.0(1.3 \text { to } 4.1)\end{array}$ & $\begin{array}{c}\text { ORR: } \\
\text { Overall: } 42 \text { (20\%; } 15 \text { to } 26) \\
\text { uninfected } \\
\text { untreated/intolerant: } 13 \\
\text { ( } 23 \% ; 13 \text { to } 36) \\
\text { uninfected Sorafenib } \\
\text { progressors: } 12(21 \% ; \\
11 \text { to } 34) \\
\text { HCV: } 10 \text { (20\%; } 10 \text { to } 34) \\
\text { HBV: } 7 \text { (14\%; } 6 \text { to } 26)\end{array}$ & Grade 3-4: (19\%) \\
\hline KEYNOTE 224 & NCT02702414 & Single-arm, CP: A & $\begin{array}{c}104 \text { pts } \\
\text { Pembrolizumab }\end{array}$ & $\begin{array}{c}\text { OS: } \\
12.9 \text { months }(95 \% \mathrm{CI} \\
9.7-15.5) \\
\text { OS at } 12 \text { months: } \\
54 \%(95 \% \text { CI } 44-63)\end{array}$ & $\begin{array}{c}\text { PFS: } \\
4.9 \text { months (95\% CI 3.4-7.2) } \\
\text { PFS at } 12 \text { months: } \\
28 \% \text { (95\% CI 19-37) }\end{array}$ & $\begin{array}{l}\text { ORR: } \\
17 \%(95 \% \text { CI 11-26) }\end{array}$ & Grade 3: $24 \%$ \\
\hline \multirow[t]{2}{*}{ He 2018} & NCT02383212 & Single-arm, CP: A & $\begin{array}{c}26 \text { pts } \\
\text { Cemiplimab }\end{array}$ & - & $\begin{array}{c}\text { PFS: } \\
3.7 \text { months (95\% CI: } 2.3-9.1)\end{array}$ & $\begin{array}{c}\text { PR: } 19.2 \% \\
\text { Stable disease: } 53.8 \%\end{array}$ & $\begin{array}{l}1 \text { death due to hepatic } \\
\text { failure related to } \\
\text { treatment }\end{array}$ \\
\hline & NCT04294498 & $\begin{array}{c}\text { Single-arm, HBV, CP: } \\
\text { A }\end{array}$ & $\begin{array}{c}43 \mathrm{pts} \\
\text { Durvalumab }\end{array}$ & - & - & - & - \\
\hline \multicolumn{8}{|c|}{ Dual immune checkpoint blockade } \\
\hline Kelley 2017 & NCT02519348 & $\begin{array}{l}\text { RCT, here we present } \\
\text { the results of initial } \\
\text { phase one safety and } \\
\text { efficacy analysis }\end{array}$ & $\begin{array}{c}40 \text { pts } \\
\text { Durvalumab/Tremelimumab } \\
\text { combination }\end{array}$ & - & - & ORR: $15 \%$ & $\begin{array}{c}\text { Most common grade } \geq 3 \\
\text { related AE was } \\
\text { asymptomatic increased } \\
\text { AST }(10 \%)\end{array}$ \\
\hline \multicolumn{8}{|c|}{ Combination with biological therapy: } \\
\hline Bang 2019 & NCT02572687 & Single-arm & $\begin{array}{c}28 \mathrm{pts} \\
\text { Ramucirumab and } \\
\text { Durvalumab }\end{array}$ & $\begin{array}{l}10.7 \text { months }(95 \% \mathrm{CI} \\
5.1-18.4)\end{array}$ & 4.4 months (95\% CI 1.6-5.7) & ORR: $3(11 \%)$ & \\
\hline Xu 2019 & NCT02942329 & Single-arm & $\begin{array}{c}18 \text { pts } \\
\text { Camrelizumab + Apatinib }\end{array}$ & OS: not reached & $\begin{array}{c}\text { PFS: } 5.8 \text { months }(2.6, \mathrm{NR}) \\
\text { At } 6 \text { months: } 45.4 \% \\
(20.9 \%, 67.1 \%) \\
\text { At } 9 \text { months: } 37.8 \% \\
(15.0 \%, 60.7 \%)\end{array}$ & ORR: $44.4 \%$ & \\
\hline
\end{tabular}

CP: Child-Pugh, RCT: Randomized Controlled Trial, RECIST: Response Evaluation Criteria in Solid Tumors, OS: Overall Survival, PFS: Progression-Free Survival, ORR: Objective Response

Rate, TTP: Time to Progression, PR: Partial Response. 


\subsection{PD-1/PD-L1 Inhibition}

The progression-free survival, overall survival and response rates were found to be better for patients treated with anti-PD-1/PD-L1 compared to placebo [25]. However, the data from the retrospective analysis did not show differences between anti-PD-1/PD-L1 when compared to regorafenib [48,49]. Interestingly, a combination of anti-PD-1/PD-L1 with radiation therapy showed better progression-free survival and overall survival when compared to anti-PD-1/PDL-1 alone [50] (Table 2).

\subsection{Dual Immune Checkpoint Blockade}

Initial results of a single-arm study examining a combination between durvalumab and tremelimumab in patients with or without hepatitis infection. Out of 40 patients treated, $6(15 \%)$ had an objective response rate with an acceptable safety profile [37] (Table 2).

\subsection{Combination with Biological Therapy}

Two studies evaluated the combination of biologic therapy with immunotherapy. The first evaluated ramucirumab plus duravalumab revealing an objective response rate of $11 \%$ and progression-free survival of 4.4 months [51]. The other one evaluated camrelizumab plus apatinib revealing an objective response rate of $44.4 \%$ and progression-free survival of 5.8 months [52] (Table 2).

\section{Predictors of Response Using PD-L1 Expression}

Immunohistochemical detection of PD-L1 has been studied in clinical trials as a predictor of response. It has been found that the expression of PD-L1 is associated with better overall response and survival outcomes [21,23]. A high tumor mutation burden (TMB), the number of somatic non-synchronous mutations in the genome of cancer cells, is a known predictive factor for response in different solid tumors. However, HCC has a low TMB compared to other solid malignancies which limited the predictive ability of this marker for HCC [53-55].

\section{Immune Checkpoint Inhibitors for Subgroups of Patients}

\subsection{Use of Immune Checkpoint Inhibitors in Patients Autoimmune Diseases}

One of the main concerns while treating patients with immune checkpoint inhibitors is immune-related adverse events which can be irreversible and even fatal [56-58]. Therefore, patients with a pre-existing auto-immune disease usually excluded from clinical trials [42], and, as a consequence, data about safety profiles in these populations is not available. However, liver cirrhosis can develop due to autoimmune diseases such as primary sclerosing cholangitis, autoimmune hepatitis, primary biliary cholangitis and so forth $[59,60]$. And, patients with HCC may suffer from another non-hepatobiliary autoimmune disease. Thus, understanding the underlying pathophysiological mechanisms and its interaction with the immune checkpoints' pathways is crucial in order to provide these patients with the therapeutic advantages without devastating side effects.

Several retrospective studies and case reports evaluated the safety profile of immune checkpoint inhibitors for patients with cancer and concomitant autoimmune disease [61-68]. Abdel-Wahab et al. conducted a systematic review evaluating the safety of immune checkpoint in patients with preexisting autoimmune disease and they found that; although some events may be severe and even fatal, most immune flares and immune-related side effects are managed without permanent drug discontinuation. However, for patients with neurological diseases such as myasthenia graves and multiple sclerosis, almost all patients developed exacerbation or immune-related side effects. Therefore, careful evaluation should be considered before prescribing immune checkpoints inhibitors for patients with neurological autoimmune diseases [61]. In a more recent large scale study, patients with a preexisting autoimmune disease treated with immune checkpoints had a higher risk of immune-related side effects than the 
control group. Furthermore, active disease and female gender were found to be independent predictors for the development of immune-related side-effects [62].

In summary, the immune-related side effects seem to be higher in patients with pre-existing autoimmune disease. Active disease and female gender are independent risk factors for immune-related side effects. Although immune-related side effects in patients treated with immune checkpoint inhibitors with a pre-existing autoimmune disease can be fatal, most cases are managed successfully without permanent discontinuation of the immune checkpoints. Nevertheless, these observations are derived from case reports and small retrospective studies and a well-designed large scale trial still represents an unmet need. Moreover, data about patients with HCC carcinoma specifically and hepatobiliary autoimmune diseases is still sparse.

\subsection{Use of Immune Checkpoint Inhibitors in Patients with Inflammatory Bowel Disease}

Patients suffering from inflammatory bowel disease (IBD) usually suffer from other hepatobiliary diseases such as drug-induced liver injury (about 30\% of patients with IBD), primary sclerosing cholangitis (1.4\% to $7.5 \%$ of patients with IBD), autoimmune hepatitis, primary biliary cirrhosis and nonalcoholic steatohepatitis [69]. These factors, along with the other traditional risk factors, can lead to HCC either directly or indirectly through liver cirrhosis [59]. Therefore, some patients who will suffer from HCC will have a concomitant IBD in which, as discussed before, immune checkpoint inhibitors with or without biologic therapy may be an option. However, the safety of immune checkpoint in this particular population is an ongoing and unanswered question. As known, the CTLA-4 and PD-1/PD-L1 signaling are crucial for gut homeostasis [70,71]. Interestingly, defects in the CTLA-4 gene or overexpression of PD-1/PD-L1 on intestinal epithelium were found to be higher in patients with IBD [72-74]. Figure 3 Therefore, in murine models, it was not surprising that the blockade of these pathways led to CD8 autoimmune enteritis [75]. And, it is not uncommon for a patient treated with immune checkpoint inhibitors to suffer from diarrhea [76]. Thus, IBD exacerbation during treatment with immune checkpoint inhibitors is a theoretical risk. Indeed, evidence about this question started to emanate from high volume centers. For example, in a recently published case series from Mayo Clinic, thirteen patients with a pre-diagnosed IBD were treated with immune checkpoint inhibitors and of them flare occurred in 4 patients (31\%) [77]. This observation was also noted in a previous cohort in which $36 \%$ of patients with IBD treated with immune checkpoint inhibitors permanently discontinued them for IBD flare [68]. From a larger sample size study, data from a multicenter retrospective analysis included 102 patients with IBD treated with immune checkpoint inhibitors. Overall gastrointestinal side effects occurred in 42 patients (41\%) after a median 62 days compared to $11 \%$ without IBD. Moreover, about $21 \%$ of patients with IBD treated with immune checkpoint inhibitors suffered from grade 3-4 diarrhea and 4 patients $(3.9 \%)$ had intestinal perforation two of them had surgery [78]. Of note, the rate of intestinal perforation in patients receiving immune checkpoint inhibitors without concomitant IBD has been reported to be about 2.2\% [79]. Importantly, most ( 90\%) of the included patients, in the aforementioned study evaluating the safety of immune checkpoints in patients with IBD, received a monotherapy of immune checkpoint inhibitors, only 10 patients $(\sim 10 \%)$ received a combination of two immune checkpoint inhibitors and none of the included patients received biologic therapy [78].

The combination of biologic therapy, especially bevacizumab, with immune checkpoint inhibitors in patients with IBD is of particular importance. Indeed, in patients not suffering from IBD, the intestinal perforation rate after using bevacizumab is about (1.5 to $2.5 \%)$ and severe bleeding is about $3 \%[80,81]$. Importantly, the mortality rate for patients who develop intestinal perforation due to bevacizumab is high (up to $16 \%$ ) [80,81]. Thus, even in non-gastrointestinal malignancies, the treatment with bevacizumab was found to be independently associated with a high risk of gastrointestinal perforation $[82,83]$. Indeed, the evidence about the safety of bevacizumab in patients with IBD is still lacking. Importantly, in patients with HCC, liver cirrhosis is common and gastrointestinal 
bleeding especially esophageal varices is a major concern, especially when selecting bevacizumab for treatment [84].

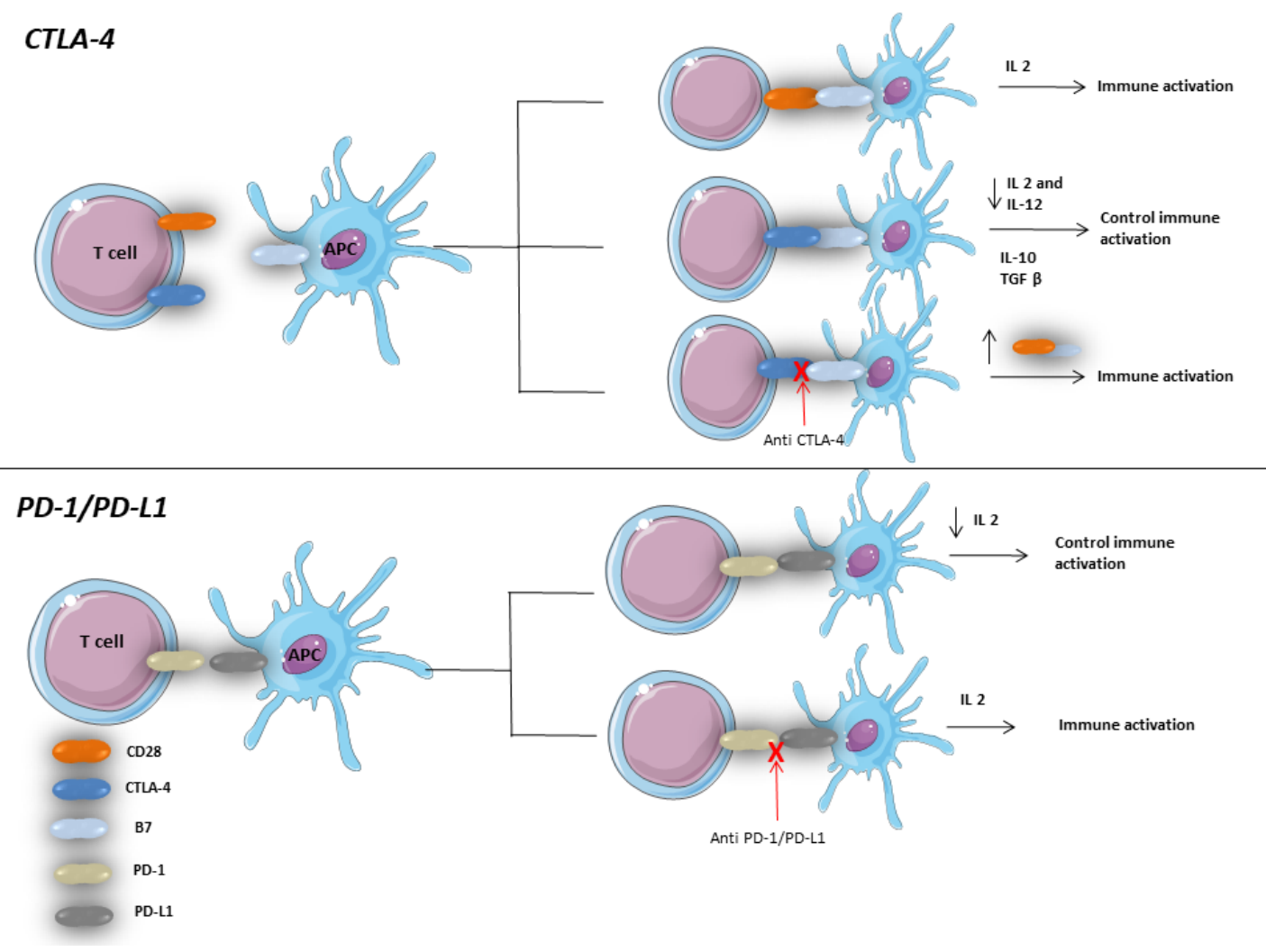

Figure 3. Role of CTLA-4 and PD-1/PD-L1 pathways in immune response regulation in gastrointestinal tract. APC: antigen-presenting cells.Both CD28 and CTLA-4 compete with each other for a binding site (B7) on the surface of APC. Binding of CD28 to B7 is associated with induction of immune response through upregulation of production of IL2. On the other side, CTLA-4 B7 binding regulates the late immune response by decreasing IL2 [71]. Therefore, inhibition of CTLA-4 by antiCTLA-4 antibodies was found to be associated with an exaggerated immune response which might lead to colitis $[85,86]$. PD-1/PD-L1 binding leads to immune response regulation through PI3K and AKT pathways. Therefore, inhibition of this binding might lead to immune response dysregulation which might lead to colitis and autoimmune exacerbation [87].

Overall, the use of atezolizumab with bevacizumab in patients with IBD carries a risk for intestinal perforation, gastrointestinal bleeding and the safety profile is still lacking in the literature.

\section{Novel Immunotherapies}

With the recent advances in the immunotherapeutic mechanisms, novel immunotherapies have gained popularity. Different therapeutic targets have been evaluated such as lymphocyte activation gene 3 (LAG-3). LAG-3 is first described by Triebel et al. and thereafter it was found to be overexpressed on the activated $\mathrm{T}$ cytotoxic and T regulatory cells with a negative impact on $\mathrm{T}$ helper cells. Therefore, during tumorigenesis, cancer cells use this pathway to escape from the immune system. Therefore, immunoglobin against LAG-3 has been investigated in different clinical trials [88]. Several other novel targets including T cell immunoglobulin and ITIM domain (TIGIT), T cell immunoglobulin and mucin domain-containing -3 (TIM-3) and B and T lymphocyte attenuator (BTLA) have been evaluated in clinical trials [88]. The current ongoing phase II trial [NCT03680508] is designed to evaluate the efficacy of TIM-3 in combination with PD-1 antibody for patients with HCC with no results published yet. 
One other therapeutic target is the killer immunoglobulin-like receptor (KIR) which has an inhibitory effect on the NK cells. Therefore, Lirilumab, a KIR antibody, is under investigation in combination with immune checkpoint inhibitors in clinical trials [89].

Overall, the novel immunotherapies' investigation in HCC is still restricted to being a part of evaluation of their role in solid tumors in general. Therefore, a better understanding of these pathways and their contribution to the HCC microenvironment is needed.

\section{Conclusions}

Hepatocellular carcinoma treatment represents a real challenge in patients with cirrhosis and several pharmacological [72-75] and loco-regional [76-81] therapies have been tested with mixed results. A combination of immune checkpoint inhibitors with biologic therapy seems to be promising for a new therapeutic standard of care for patients with unresectable HCC. However, for the subset of patients such as patients with preexisting autoimmune disease, inflammatory bowel disease or nonalcoholic steatohepatitis, the safety and efficacy are still not well established and further studies are needed to address all these open unanswered questions.

Author Contributions: Conceptualization, M.A.A.E.A. and A.F.; methodology, M.A.A.E.A. and T.N.; software, S.S.; validation, A.F., M.E. and C.C.; formal analysis, A.F.; investigation, M.A.A.E.A.; resources, R.S.; data curation, A.F.; writing —original draft preparation, A.F.; writing—review and editing, A.F. and M.A.A.E.A.; visualization, C.C.; supervision, R.S.; project administration, R.S.; funding acquisition, R.S. All authors have read and agreed to the published version of the manuscript.

Funding: This research received no external funding.

Conflicts of Interest: The authors declare no conflict of interest.

\section{References}

1. El-Serag, H.B. Hepatocellular carcinoma. N. Engl. J. Med. 2011, 365, 1118-1127. [CrossRef] [PubMed]

2. Abd El Aziz, M.A.; Sacco, R.; Facciorusso, A. Nucleos(t)ide analogues and Hepatitis B virus-related hepatocellular carcinoma: A literature review. Antivir. Chem. Chemother. 2020, 28, 2040206620921331. [CrossRef] [PubMed]

3. Kim, D.; Li, A.A.; Perumpail, B.J.; Gadiparthi, C.; Kim, W.; Cholankeril, G.; Glenn, J.S.; Harrison, S.A.; Younossi, Z.M.; Ahmed, A. Changing Trends in Etiology-Based and Ethnicity-Based Annual Mortality Rates of Cirrhosis and Hepatocellular Carcinoma in the United States. Hepatology 2019, 69, 1064-1074. [CrossRef] [PubMed]

4. Facciorusso, A.; Abd El Aziz, M.A.; Singh, S.; Pusceddu, S.; Milione, M.; Giacomelli, L.; Sacco, R. Statin Use Decreases the Incidence of Hepatocellular Carcinoma: An Updated Meta-Analysis. Cancers 2020, $12,874$. [CrossRef] [PubMed]

5. Kudo, M.; Trevisani, F.; Abou-Alfa, G.K.; Rimassa, L. Hepatocellular Carcinoma: Therapeutic Guidelines and Medical Treatment. Liver Cancer 2016, 6, 16-26. [CrossRef] [PubMed]

6. Llovet, J.M.; Ricci, S.; Mazzaferro, V.; Hilgard, P.; Gane, E.; Blanc, J.F.; de Oliveira, A.C.; Santoro, A.; Raoul, J.L.; Forner, A.; et al. Sorafenib in advanced hepatocellular carcinoma. N. Engl. J. Med. 2008, 359, 378-390. [CrossRef] [PubMed]

7. Wilhelm, S.M.; Adnane, L.; Newell, P.; Villanueva, A.; Llovet, J.M.; Lynch, M. Preclinical overview of sorafenib, a multikinase inhibitor that targets both Raf and VEGF and PDGF receptor tyrosine kinase signaling. Mol. Cancer Ther. 2008, 7, 3129-3140. [CrossRef]

8. Keating, G.M.; Santoro, A. Sorafenib. Drugs 2009, 69, 223-240. [CrossRef]

9. Kudo, M.; Finn, R.S.; Qin, S.; Han, K.H.; Ikeda, K.; Piscaglia, F.; Baron, A.; Park, J.W.; Han, G.; Jassem, J.; et al. Lenvatinib versus sorafenib in first-line treatment of patients with unresectable hepatocellular carcinoma: A randomised phase 3 non-inferiority trial. Lancet 2018, 391, 1163-1173. [CrossRef]

10. Matsui, J.; Funahashi, Y.; Uenaka, T.; Watanabe, T.; Tsuruoka, A.; Asada, M. Multi-kinase inhibitor E7080 suppresses lymph node and lung metastases of human mammary breast tumor MDA-MB-231 via inhibition of vascular endothelial growth factor-receptor (VEGF-R) 2 and VEGF-R3 kinase. Clin. Cancer Res. 2008, 14, 5459-5465. [CrossRef] 
11. Okamoto, K.; Kodama, K.; Takase, K.; Sugi, N.H.; Yamamoto, Y.; Iwata, M.; Tsuruoka, A. Antitumor activities of the targeted multi-tyrosine kinase inhibitor lenvatinib (E7080) against RET gene fusion-driven tumor models. Cancer Lett. 2013, 340, 97-103. [CrossRef]

12. Matsui, J.; Yamamoto, Y.; Funahashi, Y.; Tsuruoka, A.; Watanabe, T.; Wakabayashi, T.; Uenaka, T.; Asada, M. E7080, a novel inhibitor that targets multiple kinases, has potent antitumor activities against stem cell factor producing human small cell lung cancer H146, based on angiogenesis inhibition. Int. J. Cancer 2008, 122, 664-671. [CrossRef] [PubMed]

13. Yamamoto, Y.; Matsui, J.; Matsushima, T.; Obaishi, H.; Miyazaki, K.; Nakamura, K.; Tohyama, O.; Semba, T.; Yamaguchi, A.; Hoshi, S.S.; et al. Lenvatinib, an angiogenesis inhibitor targeting VEGFR/FGFR, shows broad antitumor activity in human tumor xenograft models associated with microvessel density and pericyte coverage. Vasc. Cell 2014, 6, 18. [CrossRef]

14. Facciorusso, A.; Abd El Aziz, M.A.; Sacco, R. Efficacy of Regorafenib in Hepatocellular Carcinoma Patients: A Systematic Review and Meta-Analysis. Cancers 2019, 12, 36. [CrossRef] [PubMed]

15. Pinter, M.; Peck-Radosavljevic, M. Review article: Systemic treatment of hepatocellular carcinoma. Aliment. Pharmacol. Ther. 2018, 48, 598-609. [CrossRef] [PubMed]

16. Bruix, J.; Qin, S.; Merle, P.; Granito, A.; Huang, Y.H.; Bodoky, G.; Pracht, M.; Yokosuka, O.; Rosmorduc, O.; Breder, V.; et al. Regorafenib for patients with hepatocellular carcinoma who progressed on sorafenib treatment (RESORCE): A randomised, double-blind, placebo-controlled, phase 3 trial. Lancet 2017, 389, 56-66. [CrossRef]

17. Abou-Alfa, G.K.; Meyer, T.; Cheng, A.-L.; El-Khoueiry, A.B.; Rimassa, L.; Ryoo, B.-Y.; Cicin, I.; Merle, P.; Chen, Y.; Park, J.-W.; et al. Cabozantinib in Patients with Advanced and Progressing Hepatocellular Carcinoma. N. Engl. J. Med. 2018, 379, 54-63. [CrossRef]

18. Zhu, A.X.; Kang, Y.K.; Yen, C.J.; Finn, R.S.; Galle, P.R.; Llovet, J.M.; Assenat, E.; Brandi, G.; Pracht, M.; Lim, H.Y.; et al. Ramucirumab after sorafenib in patients with advanced hepatocellular carcinoma and increased $\alpha$-fetoprotein concentrations (REACH-2): A randomised, double-blind, placebo-controlled, phase 3 trial. Lancet Oncol. 2019, 20, 282-296. [CrossRef]

19. Yu, S.; Wang, Y.; Hou, J.; Li, W.; Wang, X.; Xiang, L.; Tan, D.; Wang, W.; Jiang, L.; Claret, F.X.; et al. Tumor-infiltrating immune cells in hepatocellular carcinoma: Tregs is correlated with poor overall survival. PLoS ONE 2020, 15, e0231003. [CrossRef]

20. Pinato, D.J.; Guerra, N.; Fessas, P.; Murphy, R.; Mineo, T.; Mauri, F.A.; Mukherjee, S.K.; Thursz, M.; Wong, C.N.; Sharma, R.; et al. Immune-based therapies for hepatocellular carcinoma. Oncogene 2020, 39, 3620-3637. [CrossRef]

21. El-Khoueiry, A.B.; Sangro, B.; Yau, T.; Crocenzi, T.S.; Kudo, M.; Hsu, C.; Kim, T.Y.; Choo, S.P.; Trojan, J.; Welling, T.H.R.; et al. Nivolumab in patients with advanced hepatocellular carcinoma (CheckMate 040): An open-label, non-comparative, phase 1/2 dose escalation and expansion trial. Lancet 2017, 389, 2492-2502. [CrossRef]

22. Yau, T.; Kang, Y.-K.; Kim, T.-Y.; El-Khoueiry, A.B.; Santoro, A.; Sangro, B.; Melero, I.; Kudo, M.; Hou, M.-M.; Matilla, A.; et al. Nivolumab (NIVO) + ipilimumab (IPI) combination therapy in patients (pts) with advanced hepatocellular carcinoma (aHCC): Results from CheckMate 040. J. Clin. Oncol. 2019, 37, 4012. [CrossRef]

23. Zhu, A.X.; Finn, R.S.; Edeline, J.; Cattan, S.; Ogasawara, S.; Palmer, D.; Verslype, C.; Zagonel, V.; Fartoux, L.; Vogel, A.; et al. Pembrolizumab in patients with advanced hepatocellular carcinoma previously treated with sorafenib (KEYNOTE-224): A non-randomised, open-label phase 2 trial. Lancet Oncol. 2018, 19, 940-952. [CrossRef]

24. Yau, T.; Park, J.W.; Finn, R.S.; Cheng, A.L.; Mathurin, P.; Edeline, J.; Kudo, M.; Han, K.H.; Harding, J.J.; Merle, P.; et al. CheckMate 459: A randomized, multi-center phase III study of nivolumab (NIVO) vs sorafenib (SOR) as first-line (1L) treatment in patients (pts) with advanced hepatocellular carcinoma (aHCC). Ann. Oncol. 2019, 30, v874-v875. [CrossRef]

25. Finn, R.S.; Ryoo, B.Y.; Merle, P.; Kudo, M.; Bouattour, M.; Lim, H.Y.; Breder, V.; Edeline, J.; Chao, Y.; Ogasawara, S.; et al. Pembrolizumab As Second-Line Therapy in Patients With Advanced Hepatocellular Carcinoma in KEYNOTE-240: A Randomized, Double-Blind, Phase III Trial. J. Clin. Oncol. Off. J. Am. Soc. Clin. Oncol. 2020, 38, 193-202. [CrossRef] [PubMed]

26. Hanahan, D.; Weinberg, R.A. Hallmarks of cancer: The next generation. Cell 2011, 144, 646-674. [CrossRef] 
27. Khan, H.; Gucalp, R.; Shapira, I. Evolving Concepts: Immunity in Oncology from Targets to Treatments. J. Oncol. 2015, 2015, 847383. [CrossRef]

28. Shi, F.; Shi, M.; Zeng, Z.; Qi, R.Z.; Liu, Z.W.; Zhang, J.Y.; Yang, Y.P.; Tien, P.; Wang, F.S. PD-1 and PD-L1 upregulation promotes CD8(+) T-cell apoptosis and postoperative recurrence in hepatocellular carcinoma patients. Int. J. Cancer 2011, 128, 887-896. [CrossRef]

29. Gao, Q.; Wang, X.Y.; Qiu, S.J.; Yamato, I.; Sho, M.; Nakajima, Y.; Zhou, J.; Li, B.Z.; Shi, Y.H.; Xiao, Y.S.; et al. Overexpression of PD-L1 significantly associates with tumor aggressiveness and postoperative recurrence in human hepatocellular carcinoma. Clin. Cancer Res. 2009, 15, 971-979. [CrossRef]

30. Zhang, T.; Song, X.; Xu, L.; Ma, J.; Zhang, Y.; Gong, W.; Zhang, Y.; Zhou, X.; Wang, Z.; Wang, Y.; et al. The binding of an anti-PD-1 antibody to Fc $\gamma$ RI has a profound impact on its biological functions. Cancer Immunol. Immunother. CII 2018, 67, 1079-1090. [CrossRef]

31. Qin, S.; Finn, R.S.; Kudo, M.; Meyer, T.; Vogel, A.; Ducreux, M.; Macarulla, T.M.; Tomasello, G.; Boisserie, F.; Hou, J.; et al. RATIONALE 301 study: Tislelizumab versus sorafenib as first-line treatment for unresectable hepatocellular carcinoma. Future Oncol. 2019, 15, 1811-1822. [CrossRef] [PubMed]

32. Iwai, Y.; Hamanishi, J.; Chamoto, K.; Honjo, T. Cancer immunotherapies targeting the PD-1 signaling pathway. J. Biomed. Sci. 2017, 24, 26. [CrossRef] [PubMed]

33. Kudo, M. Combination Cancer Immunotherapy with Molecular Targeted Agents/Anti-CTLA-4 Antibody for Hepatocellular Carcinoma. Liver Cancer 2019, 8, 1-11. [CrossRef]

34. Wing, K.; Onishi, Y.; Prieto-Martin, P.; Yamaguchi, T.; Miyara, M.; Fehervari, Z.; Nomura, T.; Sakaguchi, S. CTLA-4 control over Foxp3+ regulatory T cell function. Science 2008, 322, 271-275. [CrossRef]

35. Kudo, M. Immuno-Oncology Therapy for Hepatocellular Carcinoma: Current Status and Ongoing Trials. Liver Cancer 2019, 8, 221-238. [CrossRef]

36. Postow, M.A.; Chesney, J.; Pavlick, A.C.; Robert, C.; Grossmann, K.; McDermott, D.; Linette, G.P.; Meyer, N.; Giguere, J.K.; Agarwala, S.S.; et al. Nivolumab and ipilimumab versus ipilimumab in untreated melanoma. N. Engl. J. Med. 2015, 372, 2006-2017. [CrossRef]

37. Kelley, R.K.; Abou-Alfa, G.K.; Bendell, J.C.; Kim, T.-Y.; Borad, M.J.; Yong, W.-P.; Morse, M.; Kang, Y.-K.; Rebelatto, M.; Makowsky, M.; et al. Phase I/II study of durvalumab and tremelimumab in patients with unresectable hepatocellular carcinoma (HCC): Phase I safety and efficacy analyses. J. Clin. Oncol. 2017, 35, 4073. [CrossRef]

38. Morse, M.A.; Sun, W.; Kim, R.; He, A.R.; Abada, P.B.; Mynderse, M.; Finn, R.S. The Role of Angiogenesis in Hepatocellular Carcinoma. Clin. Cancer Res. Off. J. Am. Assoc. Cancer Res. 2019, 25, 912-920. [CrossRef] [PubMed]

39. Zhu, A.X.; Duda, D.G.; Sahani, D.V.; Jain, R.K. HCC and angiogenesis: Possible targets and future directions. Nat. Rev. Clin. Oncol. 2011, 8, 292-301. [CrossRef] [PubMed]

40. Voron, T.; Marcheteau, E.; Pernot, S.; Colussi, O.; Tartour, E.; Taieb, J.; Terme, M. Control of the immune response by pro-angiogenic factors. Front. Oncol. 2014, 4, 70. [CrossRef]

41. Fukumura, D.; Kloepper, J.; Amoozgar, Z.; Duda, D.G.; Jain, R.K. Enhancing cancer immunotherapy using antiangiogenics: Opportunities and challenges. Nat. Rev. Clin. Oncol. 2018, 15, 325-340. [CrossRef] [PubMed]

42. Finn, R.S.; Qin, S.; Ikeda, M.; Galle, P.R.; Ducreux, M.; Kim, T.Y.; Kudo, M.; Breder, V.; Merle, P.; Kaseb, A.O.; et al. Atezolizumab plus Bevacizumab in Unresectable Hepatocellular Carcinoma. N. Engl. J. Med. 2020, 382, 1894-1905. [CrossRef]

43. Lee, M.S.; Ryoo, B.Y.; Hsu, C.H.; Numata, K.; Stein, S.; Verret, W.; Hack, S.P.; Spahn, J.; Liu, B.; Abdullah, H.; et al. Atezolizumab with or without bevacizumab in unresectable hepatocellular carcinoma (GO30140): An open-label, multicentre, phase 1b study. Lancet Oncol. 2020, 21, 808-820. [CrossRef]

44. Pinter, M.; Ulbrich, G.; Sieghart, W.; Kölblinger, C.; Reiberger, T.; Li, S.; Ferlitsch, A.; Müller, C.; Lammer, J.; Peck-Radosavljevic, M. Hepatocellular Carcinoma: A Phase II Randomized Controlled Double-Blind Trial of Transarterial Chemoembolization in Combination with Biweekly Intravenous Administration of Bevacizumab or a Placebo. Radiology 2015, 277, 903-912. [CrossRef] [PubMed]

45. Siegel, A.B.; Cohen, E.I.; Ocean, A.; Lehrer, D.; Goldenberg, A.; Knox, J.J.; Chen, H.; Clark-Garvey, S.; Weinberg, A.; Mandeli, J.; et al. Phase II trial evaluating the clinical and biologic effects of bevacizumab in unresectable hepatocellular carcinoma. J. Clin. Oncol. Off. J. Am. Soc. Clin. Oncol. 2008, 26, 2992-2998. [CrossRef] 
46. Sangro, B.; Gomez-Martin, C.; de la Mata, M.; Iñarrairaegui, M.; Garralda, E.; Barrera, P.; Riezu-Boj, J.I.; Larrea, E.; Alfaro, C.; Sarobe, P.; et al. A clinical trial of CTLA-4 blockade with tremelimumab in patients with hepatocellular carcinoma and chronic hepatitis C. J. Hepatol. 2013, 59, 81-88. [CrossRef]

47. Duffy, A.G.; Ulahannan, S.V.; Makorova-Rusher, O.; Rahma, O.; Wedemeyer, H.; Pratt, D.; Davis, J.L.; Hughes, M.S.; Heller, T.; ElGindi, M.; et al. Tremelimumab in combination with ablation in patients with advanced hepatocellular carcinoma. J. Hepatol. 2017, 66, 545-551. [CrossRef]

48. Choi, W.M.; Choi, J.; Lee, D.; Shim, J.H.; Lim, Y.S.; Lee, H.C.; Chung, Y.H.; Lee, Y.S.; Park, S.R.; Ryu, M.H.; et al. Regorafenib Versus Nivolumab After Sorafenib Failure: Real-World Data in Patients With Hepatocellular Carcinoma. Hepatol. Commun. 2020, 4, 1073-1086. [CrossRef]

49. Lee, C.H.; Lee, Y.B.; Kim, M.A.; Jang, H.; Oh, H.; Kim, S.W.; Cho, E.J.; Lee, K.H.; Lee, J.H.; Yu, S.J.; et al. Effectiveness of nivolumab versus regorafenib in hepatocellular carcinoma patients who failed sorafenib treatment. Clin. Mol. Hepatol. 2020, 26, 328-339. [CrossRef] [PubMed]

50. Yu, J.I.; Lee, S.J.; Lee, J.; Lim, H.Y.; Paik, S.W.; Yoo, G.S.; Choi, C.; Park, H.C. Clinical significance of radiotherapy before and/or during nivolumab treatment in hepatocellular carcinoma. Cancer Med. 2019, 8, 6986-6994. [CrossRef]

51. Bang, Y.-J.; Golan, T.; Lin, C.-C.; Dahan, L.; Fu, S.; Moreno, V.; Geva, R.; Reck, M.; Wasserstrom, H.A.; Mi, G.; et al. Ramucirumab (Ram) and durvalumab (Durva) treatment of metastatic non-small cell lung cancer (NSCLC), gastric/gastroesophageal junction (G/GEJ) adenocarcinoma, and hepatocellular carcinoma (HCC) following progression on systemic treatment(s). J. Clin. Oncol. 2019, 37, 2528. [CrossRef]

52. Xu, J.; Zhang, Y.; Jia, R.; Yue, C.; Chang, L.; Liu, R.; Zhang, G.; Zhao, C.; Zhang, Y.; Chen, C.; et al. Anti-PD-1 Antibody SHR-1210 Combined with Apatinib for Advanced Hepatocellular Carcinoma, Gastric, or Esophagogastric Junction Cancer: An Open-label, Dose Escalation and Expansion Study. Clin. Cancer Res. Off. J. Am. Assoc. Cancer Res. 2019, 25, 515-523. [CrossRef] [PubMed]

53. Totoki, Y.; Tatsuno, K.; Covington, K.R.; Ueda, H.; Creighton, C.J.; Kato, M.; Tsuji, S.; Donehower, L.A.; Slagle, B.L.; Nakamura, H.; et al. Trans-ancestry mutational landscape of hepatocellular carcinoma genomes. Nat. Genet. 2014, 46, 1267-1273. [CrossRef] [PubMed]

54. Yarchoan, M.; Hopkins, A.; Jaffee, E.M. Tumor Mutational Burden and Response Rate to PD-1 Inhibition. N. Engl. J. Med. 2017, 377, 2500-2501. [CrossRef]

55. Zucman-Rossi, J.; Villanueva, A.; Nault, J.C.; Llovet, J.M. Genetic Landscape and Biomarkers of Hepatocellular Carcinoma. Gastroenterology 2015, 149, 1226-1239.e1224. [CrossRef]

56. Haanen, J.; Carbonnel, F.; Robert, C.; Kerr, K.M.; Peters, S.; Larkin, J.; Jordan, K. Management of toxicities from immunotherapy: ESMO Clinical Practice Guidelines for diagnosis, treatment and follow-up. Ann. Oncol. Off. J. Eur. Soc. Med. Oncol. 2017, 28, iv119-iv142. [CrossRef] [PubMed]

57. Konstantina, T.; Konstantinos, R.; Anastasios, K.; Anastasia, M.; Eleni, L.; Ioannis, S.; Sofia, A.; Dimitris, M. Fatal adverse events in two thymoma patients treated with anti-PD-1 immune check point inhibitor and literature review. Lung Cancer 2019, 135, 29-32. [CrossRef]

58. Krenn, M.; Grisold, A.; Wohlfarth, P.; Rath, J.; Cetin, H.; Koneczny, I.; Zimprich, F. Pathomechanisms and Clinical Implications of Myasthenic Syndromes Exacerbated and Induced by Medical Treatments. Front. Mol. Neurosci. 2020, 13, 156. [CrossRef]

59. Herbst, D.A.; Reddy, K.R. Risk factors for hepatocellular carcinoma. Clin. Liver Dis. 2012, 1, $180-182$. [CrossRef]

60. Tansel, A.; Katz, L.H.; El-Serag, H.B.; Thrift, A.P.; Parepally, M.; Shakhatreh, M.H.; Kanwal, F. Incidence and Determinants of Hepatocellular Carcinoma in Autoimmune Hepatitis: A Systematic Review and Meta-analysis. Clin. Gastroenterol. Hepatol. Off. Clin. Pract. J. Am. Gastroenterol. Assoc. 2017, 15, 1207-1217.e1204. [CrossRef]

61. Abdel-Wahab, N.; Shah, M.; Lopez-Olivo, M.A.; Suarez-Almazor, M.E. Use of Immune Checkpoint Inhibitors in the Treatment of Patients With Cancer and Preexisting Autoimmune Disease: A Systematic Review. Ann. Intern. Med. 2018, 168, 121-130. [CrossRef] [PubMed]

62. Cortellini, A.; Buti, S.; Santini, D.; Perrone, F.; Giusti, R.; Tiseo, M.; Bersanelli, M.; Michiara, M.; Grassadonia, A.; Brocco, D.; et al. Clinical Outcomes of Patients with Advanced Cancer and Pre-Existing Autoimmune Diseases Treated with Anti-Programmed Death-1 Immunotherapy: A Real-World Transverse Study. Oncologist 2019, 24, e327-e337. [CrossRef] [PubMed] 
63. Danlos, F.X.; Voisin, A.L.; Dyevre, V.; Michot, J.M.; Routier, E.; Taillade, L.; Champiat, S.; Aspeslagh, S.; Haroche, J.; Albiges, L.; et al. Safety and efficacy of anti-programmed death 1 antibodies in patients with cancer and pre-existing autoimmune or inflammatory disease. Eur. J. Cancer 2018, 91, 21-29. [CrossRef] [PubMed]

64. Johnson, D.B.; Sullivan, R.J.; Ott, P.A.; Carlino, M.S.; Khushalani, N.I.; Ye, F.; Guminski, A.; Puzanov, I.; Lawrence, D.P.; Buchbinder, E.I.; et al. Ipilimumab Therapy in Patients With Advanced Melanoma and Preexisting Autoimmune Disorders. JAMA Oncol. 2016, 2, 234-240. [CrossRef]

65. Kähler, K.C.; Eigentler, T.K.; Gesierich, A.; Heinzerling, L.; Loquai, C.; Meier, F.; Meiss, F.; Pföhler, C.; Schlaak, M.; Terheyden, P.; et al. Ipilimumab in metastatic melanoma patients with pre-existing autoimmune disorders. Cancer Immunol. Immunother. CII 2018, 67, 825-834. [CrossRef] [PubMed]

66. Leonardi, G.C.; Gainor, J.F.; Altan, M.; Kravets, S.; Dahlberg, S.E.; Gedmintas, L.; Azimi, R.; Rizvi, H.; Riess, J.W.; Hellmann, M.D.; et al. Safety of Programmed Death-1 Pathway Inhibitors Among Patients With Non-Small-Cell Lung Cancer and Preexisting Autoimmune Disorders. J. Clin. Oncol. Off. J. Am. Soc. Clin. Oncol. 2018, 36, 1905-1912. [CrossRef]

67. Menzies, A.M.; Johnson, D.B.; Ramanujam, S.; Atkinson, V.G.; Wong, A.N.M.; Park, J.J.; McQuade, J.L.; Shoushtari, A.N.; Tsai, K.K.; Eroglu, Z.; et al. Anti-PD-1 therapy in patients with advanced melanoma and preexisting autoimmune disorders or major toxicity with ipilimumab. Ann. Oncol. Off. J. Eur. Soc. Med. Oncol. 2017, 28, 368-376. [CrossRef]

68. Tison, A.; Quéré, G.; Misery, L.; Funck-Brentano, E.; Danlos, F.X.; Routier, E.; Robert, C.; Loriot, Y.; Lambotte, O.; Bonniaud, B.; et al. Safety and Efficacy of Immune Checkpoint Inhibitors in Patients With Cancer and Preexisting Autoimmune Disease: A Nationwide, Multicenter Cohort Study. Arthritis Rheumatol. 2019, 71, 2100-2111. [CrossRef]

69. Rojas-Feria, M.; Castro, M.; Suárez, E.; Ampuero, J.; Romero-Gómez, M. Hepatobiliary manifestations in inflammatory bowel disease: The gut, the drugs and the liver. World J. Gastroenterol. 2013, 19, 7327-7340. [CrossRef]

70. Dougan, M. Checkpoint Blockade Toxicity and Immune Homeostasis in the Gastrointestinal Tract. Front. Immunol. 2017, 8, 1547. [CrossRef]

71. Chambers, C.A.; Allison, J.P. Costimulatory regulation of T cell function. Curr. Opin. Cell Biol. 1999, 11, 203-210. [CrossRef]

72. Ng, S.C.; Tsoi, K.K.; Kamm, M.A.; Xia, B.; Wu, J.; Chan, F.K.; Sung, J.J. Genetics of inflammatory bowel disease in Asia: Systematic review and meta-analysis. Inflamm. Bowel Dis. 2012, 18, 1164-1176. [CrossRef]

73. Nakazawa, A.; Dotan, I.; Brimnes, J.; Allez, M.; Shao, L.; Tsushima, F.; Azuma, M.; Mayer, L. The expression and function of costimulatory molecules B7H and B7-H1 on colonic epithelial cells. Gastroenterology 2004, 126, 1347-1357. [CrossRef]

74. Beswick, E.J.; Grim, C.; Singh, A.; Aguirre, J.E.; Tafoya, M.; Qiu, S.; Rogler, G.; McKee, R.; Samedi, V.; Ma, T.Y.; et al. Expression of Programmed Death-Ligand 1 by Human Colonic CD90(+) Stromal Cells Differs Between Ulcerative Colitis and Crohn's Disease and Determines Their Capacity to Suppress Th1 Cells. Front. Immunol. 2018, 9, 1125. [CrossRef] [PubMed]

75. Reynoso, E.D.; Elpek, K.G.; Francisco, L.; Bronson, R.; Bellemare-Pelletier, A.; Sharpe, A.H.; Freeman, G.J.; Turley, S.J. Intestinal tolerance is converted to autoimmune enteritis upon PD-1 ligand blockade. J. Immunol. 2009, 182, 2102-2112. [CrossRef] [PubMed]

76. Boutros, C.; Tarhini, A.; Routier, E.; Lambotte, O.; Ladurie, F.L.; Carbonnel, F.; Izzeddine, H.; Marabelle, A.; Champiat, S.; Berdelou, A.; et al. Safety profiles of anti-CTLA-4 and anti-PD-1 antibodies alone and in combination. Nat. Rev. Clin. Oncol. 2016, 13, 473-486. [CrossRef] [PubMed]

77. Braga Neto, M.B.; Ramos, G.P.; Loftus, E.V., Jr.; Faubion, W.A.; Raffals, L.E. Use of Immune Checkpoint Inhibitors in Patients With Pre-established Inflammatory Bowel Diseases: Retrospective Case Series. Clin. Gastroenterol. Hepatol. Off. Clin. Pract. J. Am. Gastroenterol. Assoc. 2020. [CrossRef]

78. Abu-Sbeih, H.; Faleck, D.M.; Ricciuti, B.; Mendelsohn, R.B.; Naqash, A.R.; Cohen, J.V.; Sellers, M.C.; Balaji, A.; Ben-Betzalel, G.; Hajir, I.; et al. Immune Checkpoint Inhibitor Therapy in Patients With Preexisting Inflammatory Bowel Disease. J. Clin. Oncol. 2019, 38, 576-583. [CrossRef]

79. Abu-Sbeih, H.; Ali, F.S.; Luo, W.; Qiao, W.; Raju, G.S.; Wang, Y. Importance of endoscopic and histological evaluation in the management of immune checkpoint inhibitor-induced colitis. J. Immunother. Cancer 2018, 6, 95. [CrossRef] 
80. Hurwitz, H.; Fehrenbacher, L.; Novotny, W.; Cartwright, T.; Hainsworth, J.; Heim, W.; Berlin, J.; Baron, A.; Griffing, S.; Holmgren, E.; et al. Bevacizumab plus irinotecan, fluorouracil, and leucovorin for metastatic colorectal cancer. N. Engl. J. Med. 2004, 350, 2335-2342. [CrossRef]

81. Sugrue, M.; Kozloff, M.; Hainsworth, J.; Badarinath, S.; Cohn, A.; Flynn, P.; Steis, R.; Dong, W.; Sarkar, S.; Grothey, A. Risk factors for gastrointestinal perforations in patients with metastatic colorectal cancer receiving bevacizumab plus chemotherapy. J. Clin. Oncol. 2006, 24, 3535. [CrossRef]

82. Wu, Y.S.; Shui, L.; Shen, D.; Chen, X. Bevacizumab combined with chemotherapy for ovarian cancer: An updated systematic review and meta-analysis of randomized controlled trials. Oncotarget 2017, 8 , 10703-10713. [CrossRef]

83. Burger, R.A.; Brady, M.F.; Bookman, M.A.; Monk, B.J.; Walker, J.L.; Homesley, H.D.; Fowler, J.; Greer, B.E.; Boente, M.; Fleming, G.F.; et al. Risk factors for GI adverse events in a phase III randomized trial of bevacizumab in first-line therapy of advanced ovarian cancer: A Gynecologic Oncology Group Study. J. Clin. Oncol. Off. J. Am. Soc. Clin. Oncol. 2014, 32, 1210-1217. [CrossRef] [PubMed]

84. Fang, P.; Hu, J.H.; Cheng, Z.G.; Liu, Z.F.; Wang, J.L.; Jiao, S.C. Efficacy and safety of bevacizumab for the treatment of advanced hepatocellular carcinoma: A systematic review of phase II trials. PLoS ONE 2012, 7, e49717. [CrossRef] [PubMed]

85. Read, S.; Malmström, V.; Powrie, F. Cytotoxic T lymphocyte-associated antigen 4 plays an essential role in the function of CD25(+)CD4(+) regulatory cells that control intestinal inflammation. J. Exp. Med. 2000, 192, 295-302. [CrossRef] [PubMed]

86. Karandikar, N.J.; Vanderlugt, C.L.; Walunas, T.L.; Miller, S.D.; Bluestone, J.A. CTLA-4: A negative regulator of autoimmune disease. J. Exp. Med. 1996, 184, 783-788. [CrossRef] [PubMed]

87. Francisco, L.M.; Sage, P.T.; Sharpe, A.H. The PD-1 pathway in tolerance and autoimmunity. Immunol. Rev. 2010, 236, 219-242. [CrossRef] [PubMed]

88. Qin, S.; Xu, L.; Yi, M.; Yu, S.; Wu, K.; Luo, S. Novel immune checkpoint targets: Moving beyond PD-1 and CTLA-4. Mol. Cancer 2019, 18, 155. [CrossRef]

89. Vey, N.; Dumas, P.Y.; Recher, C.; Gastaud, L.; Lioure, B.; Bulabois, C.E.; Pautas, C.; Marolleau, J.P.; Leprêtre, S.; Raffoux, E.; et al. Randomized phase 2 trial of lirilumab (anti-KIR monoclonal antibody, mab) as maintenance treatment in elderly patients (pts) with acute myeloid leukemia (AML): Results of the EFFIKIR trial. Blood $2017,130,889$.

Publisher's Note: MDPI stays neutral with regard to jurisdictional claims in published maps and institutional affiliations.

(C) 2020 by the authors. Licensee MDPI, Basel, Switzerland. This article is an open access article distributed under the terms and conditions of the Creative Commons Attribution (CC BY) license (http://creativecommons.org/licenses/by/4.0/). 